Commun. math. Phys. 26, 102-108 (1972)

(C) by Springer-Verlag 1972

\title{
Phase Transitions in Anisotropic Classical Heisenberg Ferromagnets *
}

Alfred B. Bortz and Robert B. Griffiths

Department of Physics, Carnegie-Mellon University, Pittsburgh, Pennsylvania, U.S.A.

Received January 10, 1972

\begin{abstract}
The method of Peierls is used to prove the existe ce of a spontaneous magnetization for a spin system with nearest-neighbor interactions and Hamiltonian $H=-J \sum_{\langle\imath j\rangle}\left[S_{i}^{z} S_{j}^{z}+\alpha\left(S_{\imath}^{x} S_{j}^{x}+S_{i}^{y} S_{\imath}{ }^{y}\right)\right], S_{\imath}$ a (classical) unit vector at the $i^{\prime}$ th site, with $|\alpha|<0.0298$ and 0.0198 for a square lattice and simple cubic lattice, respectively.
\end{abstract}

\section{Introduction}

In the modern theory of phase transitions an argument due, in essence, to Peierls [1], and later made rigorous by Dobrushin [2] and Griffiths [3] has been very useful for proving the existence of phase transitions in several model systems $[4,5]$. In particular Ginibre [6] (see also Robinson [13]) has considered the anisotropic Heisenberg model or quantum lattice gas with Hamiltonian

$$
\begin{aligned}
& H=U+K ; \quad U=-J \sum_{\langle i j\rangle} S_{i}{ }^{z} S_{j}{ }^{z} ; \\
& K=-J \alpha \sum_{\langle i j\rangle}\left(S_{i}^{x} S_{j}^{x}+S_{i}^{y} S_{j}^{y}\right),
\end{aligned}
$$

where $S_{i}{ }^{x}$, etc. are the angular momentum operators (Pauli matrices) for a spin $1 / 2$ atom located at a site $i$ on a regular $d$-dimensional (hyper) cubic lattice $(d \geqq 2),\langle i j\rangle$ denotes a pair of nearest neighbor sites on the lattice (each pair counted in the sum only once), $J$ is a positive constant and $\alpha$ a real number.

For this model (only one of several which he considered), Ginibre proved the existence of a phase transition - the two phases characterized by the thermal average $\left\langle S_{i}{ }^{2}\right\rangle$ having positive and negative values, respectively, in the thermodynamic limit - for sufficiently low temperatures and provided $|\alpha|$ is sufficiently small. The actual estimates employed by Ginibre (which could, no doubt, be refined) require that $|\alpha|$ not exceed $10^{-6}$ for $d=2$, with even smaller limits for larger $d$. On the other hand, Fisher [7] has given intuitive arguments which suggest that a phase transition probably occurs for any $|\alpha|<1$, while Mermin and Wagner [8]

* Research supported by the National Science Foundation Grant No. GP 11454. 
have proved that a phase transition (of the sort considered here) is impossible for $|\alpha|=1$ and $d=2$.

The arguments of Fisher and of Mermin and Wagner apply also to the corresponding "classical" spin problem in which the $S_{i}$ in (1.1) are vectors of unit length rather than quantum mechanical operators. The classical problem, which is the subject of the present paper, comes about in the limit as the spin of each atom tends to infinity [9], and is simpler than the quantum case considered by Ginibre [6] in that one need not deal with non-commuting operators. However, the introduction of continuous variables does lead to some complications.

We are able to show that the classical system has a phase transition at a sufficiently low temperature provided $|\alpha|<0.0298$ for $d=2$ and $|\alpha|<0.0198$ for $d=3$. While these values are larger than those permitted in Ginibre's argument for the spin 1/2 case, they are substantially less than one might hope to achieve. On the other hand, it seems to us that the Peierls argument, at least in the form we employ, is likely to be of little use in the vicinity of $|\alpha|=1$, for reasons stated in Sec. IV below.

\section{Notation}

The unit vector

$$
\left(S_{j}^{x}\right)^{2}+\left(S_{j}^{y}\right)^{2}+\left(S_{j}^{z}\right)^{2}=1
$$

associated with site $j$ can be described in polar coordinates with an azimuthal angle $\phi_{j}$ and a polar angle $\theta_{j}$, or by $\phi_{j}$ and

$$
\zeta_{j}=S_{j}{ }^{2}=\cos \theta_{j} \text {. }
$$

In particular, $K$ in (1.1) is of the form

$$
K=-J \alpha \sum_{\langle i j\rangle}\left[\left(1-\zeta_{i}^{2}\right)\left(1-\zeta_{j}^{2}\right)\right]^{1 / 2} \cos \left(\phi_{i}-\phi_{j}\right) .
$$

Let $\Omega$ be a finite set of sites $i=1,2, \ldots V$. Following the usual custom, we shall refer to the space of variables $\phi_{1}, \zeta_{1}, \ldots \phi_{V}, \zeta_{V}$ as the "phase space" $\Gamma$ and a particular point in the space, denoted by $\xi$, as a "configuration". The Boltzmann probability distribution is

$$
P(\xi)=e^{-\beta H_{S 2}(\xi)} / Z_{\Omega},
$$

where $\beta$ is the inverse temperature, $H_{\Omega}=U_{\Omega}+K_{\Omega}$ is obtained by restricting the sums in (1.1) to sites in $\Omega$, and the partition function is

$$
Z_{\Omega}=\int_{\Gamma} d \xi e^{-\beta H_{\Omega}}=\int_{0}^{2 \pi} d \phi_{1} \int_{-1}^{1} d \zeta_{1} \ldots \int_{0}^{2 \pi} d \phi_{V} \int_{-1}^{1} d \zeta_{V} e^{-\beta H_{S}} .
$$

Thermal averages are defined with respect to (2.3), e.g.:

$$
\left\langle S_{j}{ }^{2}\right\rangle_{\Omega}=\int_{\Gamma} d \xi \zeta_{j} e^{-\beta H_{s 2}} / Z_{\Omega} .
$$

8 Commun. math. Phys., Vol. 26 


\section{Peierls Argument}

In this section we consider a square lattice with $\Omega$ all the sites within a large square. If the sites on the boundary of the square are constrained to have $S_{i}^{z}=1$ (i.e., only configurations satisfying this condition will be considered), we shall show that under suitable conditions there is a constant $\varepsilon>0$, which depends on $J, \alpha$, and $\beta$ but is independent of the size of the square, such that for any site $j$ in the interior of the square

$$
\left\langle S_{j}{ }^{z}\right\rangle_{\Omega}=\left\langle\zeta_{j}\right\rangle_{\Omega} \geqq \varepsilon .
$$

The steps required to deduce from (3.1) the existence of a phase transition in the thermodynamic limit are but minor modifications of the usual arguments [4] and we shall not repeat them here.

Since the classical vectors we are considering vary continuously, it is necessary to modify the usual Peierls procedure for constructing borders. For a given configuration $\xi$ we shall say that a site $j$ (equivalently, the spin at the site) is in class $n=1,2,3$ provided:

$$
\begin{aligned}
& n=1: 1 / 3 \leqq \zeta_{j} \leqq 1, \\
& n=2:-1 / 3<\zeta_{j}<1 / 3, \\
& n=3:-1 \leqq \zeta_{j} \leqq-1 / 3 .
\end{aligned}
$$

One may imagine borders (Fig. 1) drawn between pairs of adjacent sites on the lattice if one site is in class 1 and the other is in class 2 or 3 . Due to the condition $\zeta_{i}=+1$ for sites on the boundary of $\Omega$, such borders are closed polygons, and it is evident that any site in class 2 or 3 falls inside at least one such polygon. For simplicity, we restrict our attention to the "outer borders" which are not enclosed inside other borders. Let $p$ be the

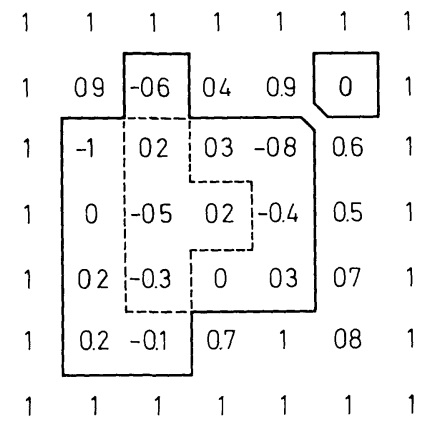

Fig. 1. Example of a configuration showing the values of $\zeta_{t}$ at each site. The border $B$ is the solid line of length 18 (in units of the lattice constant). Another border of length 4 also occurs in this configuration. The 11 sites situated between the solid line and the dashed line are the circumference sites for $B$ 
probability that the site $j$ lies inside an outer border. Since $\zeta_{j}$ is not less than $1 / 3$ if $j$ is not inside an outer border, we see that

$$
\left\langle\zeta_{j}\right\rangle \geqq \frac{1}{3}(1-p)-p,
$$

and hence (3.1) can be established by showing that $p$ is sufficiently small, less than $(1-3 \varepsilon) / 4$.

To bound $p$, we shall show that the probability $P_{B}$ of occurrence of a particular outer border $B$ (having a specified shape and position on the lattice) is bounded by

$$
P_{B} \leqq a m^{b} e^{-\beta J \gamma b},
$$

where $b$ is the length of $B$ (we assume the lattice constant is 1 ) and $a, m$, and $\gamma$ are positive constants independent of $B$. Since the number of polygons of length $b(=4,6,8$, etc.) enclosing a particular site does not exceed $b^{2} 3^{b}$, it is evident that, whatever the values of $a, m$, and $\gamma$, we can make $p$ arbitrarily small by choosing $\beta$ sufficiently large, which means a sufficiently low temperature.

Thus the only problem remaining is to establish (3.4) for

$$
P_{B}=\int_{\mathscr{B}} d \xi e^{-\beta H_{\Omega 2}} / \int_{\Gamma} d \xi e^{-\beta H_{\Omega}},
$$

where $\mathscr{B}$ denotes the set of configurations in $\Gamma$ for which the border $B$ occurs (as an outer border). The bound is obtained by introducing a mapping $\tau$ of $\mathscr{B}$ into $\Gamma$ with the following properties: (i) for $\xi \in \mathscr{B}$,

$$
H_{\Omega}(\tau \xi) \leqq H_{\Omega}(\xi)-\gamma J b
$$

(ii) the region $\mathscr{B}$ is the union of a number of disjoint regions $\mathscr{B}_{i}$, and $\tau$ maps each $\mathscr{B}_{i}$ one-to-one and continuously into a region $\mathscr{C}_{i}$ having the same phase volume (Lebesgue measure). The different regions $\mathscr{C}_{i}$ (as indicated schematically in Fig. 2) are not, in general, disjoint, but the maximum number which overlap at any point in $\Gamma$, which is to say the
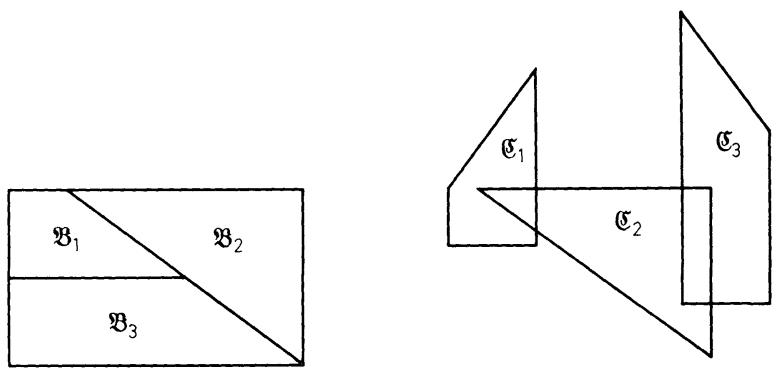

Fig. 2. Illustration (schematic) of the properties of the map $\tau$ 
maximum number of points of $\mathscr{B}$ which are mapped by $\tau$ into the same point in $\Gamma$, is bounded by $a m^{b}$. These properties combine to yield

$$
\begin{aligned}
\int_{\mathscr{B}} d \xi e^{-\beta H_{S_{2}}} & =\sum_{i} \int_{\mathscr{B}_{2}} d \xi e^{-\beta H_{\Omega_{2}}} \\
& \leqq \sum_{i} e^{-\beta J \gamma b} \int_{\mathscr{C}_{i}} d \xi e^{-\beta H_{\Omega}} \leqq a m^{b} e^{-\beta J \gamma b} \int_{\Gamma} d \xi e^{-\beta H_{\Omega_{2}}},
\end{aligned}
$$

which implies (3.4).

The map $\tau$ is constructed as follows. For brevity we adopt the term "circumference sites" to denote those sites inside $B$ which have at least one nearest-neighbor site outside $B$ (Fig. 1), that is, sites adjacent to the inside of border $B$. Let $\tau_{1}$ be the transformation which for every circumference site replaces $\zeta_{j}$ by $\zeta_{j}-2 / 3$ if $j$ is in class 2 , and leaves $\zeta_{j}$ unchanged if $j$ is in class 3 , or if $j$ is not a circumference site. (All transformations considered here leave all the $\phi_{j}$ unchanged.) Let $\tau_{r}$ be the transformation, analogous to that used in the simple Peierls argument, which changes $\zeta_{j}$ to $-\zeta_{j}$ for every site inside $B$.

If $\tau_{1}$ causes $U_{\Omega}$ to decrease, or to increase by at most $J b / 9$, that is if

$$
U_{\Omega}\left(\tau_{1} \xi\right) \leqq U_{\Omega}(\xi)+J b / 9,
$$

then $\tau$ is defined as the product $\tau_{r} \cdot \tau_{1}$. Since for $\xi \in \mathscr{B}$ every circumference site in the configuration $\tau_{1} \xi$ is in class 3 , while every site outside $B$ which is adjacent to a circumference site is in class 1 , the application of $\tau_{r}$ to $\tau_{1} \xi$ causes a decrease in $U_{\Omega}$ of at least $2 \mathrm{Jb} / 9$ :

$$
U_{\Omega}(\tau \xi)=U_{\Omega}\left(\tau_{r} \tau_{1} \xi\right) \leqq U_{\Omega}\left(\tau_{1} \xi\right)-2 J b / 9 \leqq U_{\Omega}(\xi)-J b / 9 .
$$

On the other hand if for some $\xi \in \mathscr{B}$ (3.8) is not satisfied, $\tau \xi$ is defined to be $\tau_{2} \xi$, where $\tau_{2}$ replaces $\zeta_{j}$ by $\zeta_{j}+2 / 3$ if $j$ is a circumference site in class 2 , while leaving all other $\zeta_{j}$ unchanged. It is then the case that

$$
U_{\Omega}(\tau \xi)=U_{\Omega}\left(\tau_{2} \xi\right) \leqq U_{\Omega}(\xi)-J b / 9 .
$$

This result may be obtained as follows. The change in $U_{\Omega}$ which comes about from application of $\tau_{1}$ or $\tau_{2}$ has two sources. First there are pairs of sites one of which is a circumference site in class 2 and the other a site not on the circumference or a circumference site in class 3 . The change in $U_{\Omega}$ from such pairs is evidently the same in magnitude but opposite in sign for the two transformations $\tau_{1}$ and $\tau_{2}$. A second contribution comes from adjacent sites which are both circumference sites in class 2 . The contribution of these terms to $U_{\Omega}$ invariably decreases (becomes more negative) under the application of either $\tau_{1}$ or $\tau_{2}$. Consequently if (3.8) is violated for some $\xi \in \mathscr{B},(3.10)$ must be satisfied.

The azimuthal angles $\phi_{i}$ are not altered by $\tau$, but the changes in $\zeta_{i}$ will in general change $K$. Of course $\tau_{r}$ does not affect $K$, so we need only 
consider the effects of $\tau_{1}$ and $\tau_{2}$. If $\zeta_{i}$ or $\zeta_{j}$ or both are altered by $\pm 2 / 3$ (while remaining in the allowed range $-1 \leqq \zeta \leqq 1$ ), the square root of the quantity in square brackets in (2.2) can change by at most $(8 / 9)^{1 / 2}$. In addition, if site $i$ is in class $1\left(\zeta_{i} \geqq 1 / 3\right)$ and $\zeta_{j}$ is altered by $\pm 2 / 3$, the change is at most $8 / 9$. As there are at most $b$ circumference sites and each of these has four nearest neighbors at least one of which is outside $B$ and, consequently, in class 1 , we conclude that

$$
K_{\Omega}(\tau \xi) \leqq K_{\Omega}(\xi)+|\alpha| J b\left[(8 / 9)+3(8 / 9)^{1 / 2}\right]
$$

for a square lattice. Combining this with the previous estimates, (3.9) and (3.10), for $U_{\Omega}$, we find that (3.6) is satisfied with

$$
\gamma=[1-|\alpha|(8+18 \sqrt{2})] / 9
$$

positive provided $|\alpha|<0.0298$.

The transformations $\tau_{1}$ and $\tau_{2}$ are both many-to-one. Thus in order to reconstruct $\xi$ from $\tau_{1} \xi$ it is necessary and sufficient to know at which circumference sites $\zeta_{3}$ was decreased by $2 / 3$. As there are at most $b$ circumference sites, a given $\tau_{1} \xi$ can have come from at most $2^{b}$ possible configurations in $\mathscr{B}$. The same remarks apply to $\tau_{2} \xi$, so we conclude that $\tau$ is at most $2^{b+1}$ to 1 , and thus (3.4) is satisfied with $a=m=2$. This completes the Peierls argument for the square lattice.

The extension of the Peierls argument to lattices of dimension $d>2$ is straightforward. For $d=3$ it is necessary to replace the square bracket in (3.11) with $8 / 9+5(8 / 9)^{1 / 2}$, and as a result $|\alpha|$ must not exceed 0.0198 in order to insure that $\gamma$ is positive.

\section{Concluding Remarks}

It is perhaps worth noting that the machinery developed above can be used to prove the existence of phase transitions for certain other models involving classical spins, for example:

$$
H=-J \sum_{\langle i j\rangle} S_{i}^{z} S_{j}^{z}+L \sum_{i}\left(S_{i}^{z}\right)^{2},
$$

where we assume $L>0$ as well as $J>0$, and $L / J<1 / 8$. (When $L$ is zero or negative, somewhat better results can be obtained by an entirely different route, using correlation $(G K S)$ inequalities [10]).

One wonders if our rather poor upper bounds for $|\alpha|$ may be improved. No doubt certain technical refinements of our argument are possible, but it is doubtful whether one can establish a phase transition for $|\alpha|$ less than, but close to, 1 , without a rather drastic revision of the entire Peierls procedure. The essence of the Peierls argument is the estimation of the probability of occurence of a boundary, or boundaries, separating unlike 
phases. With $\alpha$ near 1 the boundary is expected, on physical grounds $[11,12]$, to be a Bloch wall in which the spin direction alters gradually over many lattice sites. To obtain a configuration of lower energy given one in which such a Bloch wall occurs as a "border" it will be necessary to alter the configuration in a complicated way on a very large number of sites, and there seems to be no simple prescription for accomplishing this, in contrast to the usual Peierls argument which makes use of a welldefined and sharp border.

\section{References}

1. Peierls, R.: Proc. Cambridge Phil. Soc. 32, 477 (1936).

2. Dobrushin, R. L.: Teor. Veroyat. Ee Primen. 10, 209 (1965).

3. Griffiths, R. B.: Phys. Rev. 136, A 437 (1964).

4. - In: Phase transitions and critical phenomena, C.Domb and M.S.Green, eds, Vol. I. London: Academic Press 1972.

5. Ruelle, D.: Phys. Rev. Letters 27, 1040 (1971).

6. Ginibre, J.: Commun. math. Phys. 14, 205 (1969).

7. Fisher, M.E.: J. Appl. Phys. 38, 981 (1967).

8. Mermin, N.D., Wagner, H.: Phys. Rev. Letters 17, 1133 (1966).

9. Millard, K., Leff, H. S.: J. Math. Phys. 12, 1000 (1971).

10. Griffiths, R. B.: J. Math. Phys. 10, 1559 (1969).

11. Tebble, R. S.: Magnetic domains, p. 34. London: Methuen \& Co., Ltd. 1969.

12. Döring, W.: In: Flügge, S. (Ed.): Handbuch der Physik, Vol. 18/2, p. 341. BerlinHeidelberg-New York: Springer 1966.

13. Robinson, D. W.: Commun. math. Phys. 14, 195 (1969).

Alfred B. Bortz

Firelands Campus

Bowling Green State University

Huron, Ohio 44839, USA

(present address) 